\title{
Quantifying sequencing error and effective sequencing depth of liquid biopsy NGS with UMI error correction
}

\author{
Malene Støchkel Frank ${ }^{\ddagger}, 1,2$ iD, Janina Fuß ${ }^{\ddagger}, 3$ iD, Tim Alexander Steiert ${ }^{3}$, Greta Streleckiene ${ }^{4}$ iD, Julie GehI ${ }^{1,2}$ \& Michael \\ Forster*, 3 iD \\ ${ }^{1}$ Department of Clinical Oncology \& Palliative Care, Zealand University Hospital, Køge, Denmark; ${ }^{2}$ Department of Clinical Medicine, Faculty of Health \& Medical Sciences, \\ University of Copenhagen, Copenhagen, Denmark; ${ }^{3}$ Institute of Clinical Molecular Biology, Christian Albrechts University Kiel, Kiel, 24105, Germany; ${ }^{4}$ Institute for Digestive \\ Research, Lithuanian University of Health Sciences, Kaunas, 50161, Lithuania; *Author for correspondence: m.forster@ikmb.uni-kiel.de; ${ }^{\ddagger}$ Authors contributed equally
}

BioTechniques 70: 227-232 (April 2021) 10.2144/btn-2020-0124

First draft submitted: 18 August 2020; Accepted for publication: 5 January 2021; Published online: 29 January 2021

\section{ABSTRACT}

Liquid biopsies are a minimally invasive method to diagnose and longitudinally monitor tumor mutations in patients when tissue biopsies are difficult (e.g., in lung cancer). The percentage of cell-free tumor DNA in blood plasma ranges from more than $65 \%$ to $0.1 \%$ or lower. To reliably diagnose tumor mutations at $0.1 \%$, there are two options: unrealistically large volumes of patient blood or library preparation and sequencing depth optimized to low-input DNA. Here, we assess two library preparation methods and analysis workflows to determine feasibility and reliability based on standards with known allelic frequency ( 0 and $0.13 \%$ in PIK3CA). However, the implementation for patients is still costly and requires elaborate setups.

\section{GRAPHICAL ABSTRACT}
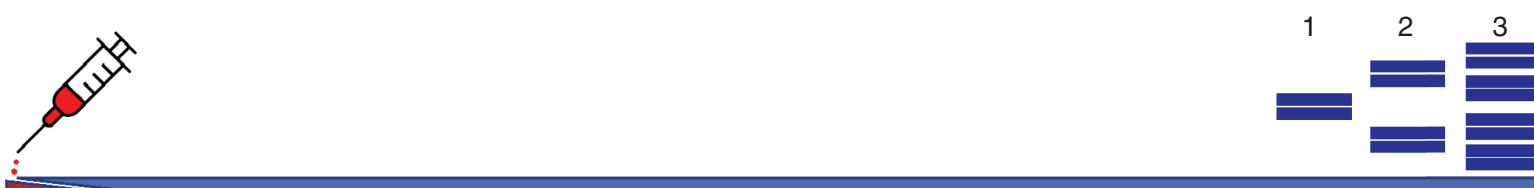

Number of PCR cycles

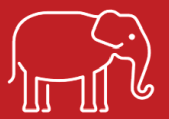

If only human input is available: higher degree of specialization \& costs

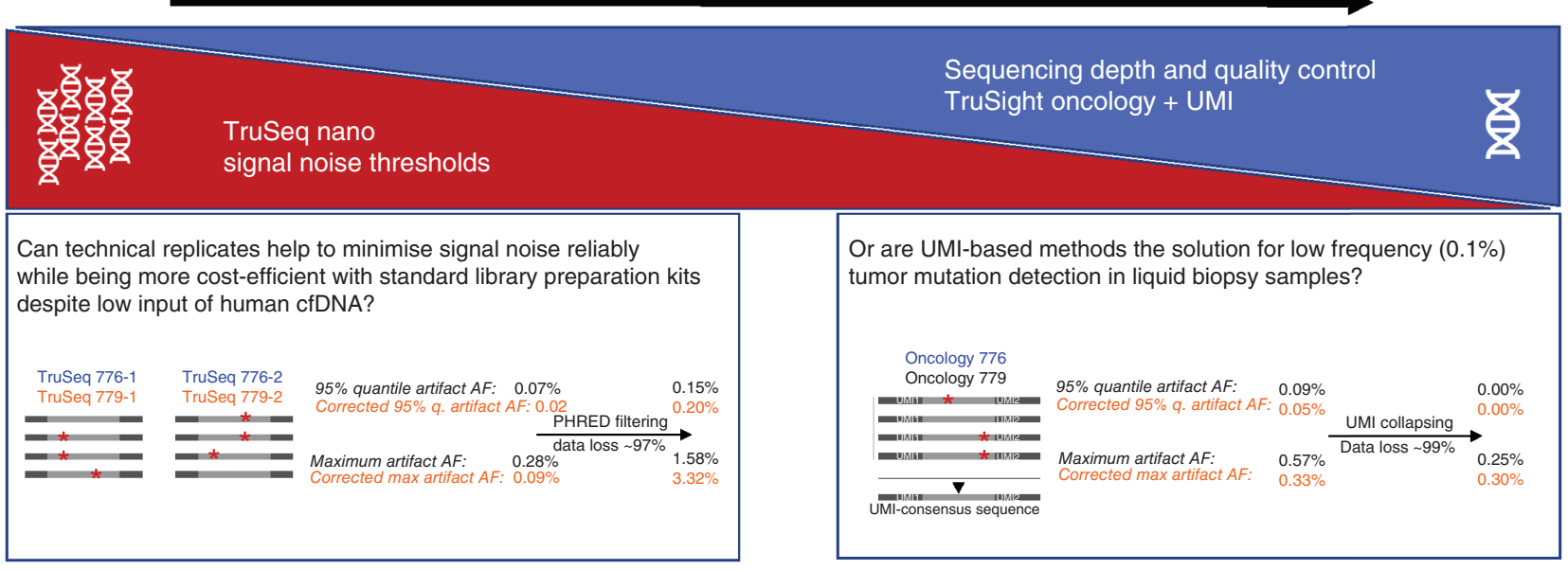




\section{METHOD SUMMARY}

Two widely used Illumina library preparation kits were benchmarked for next-generation sequencing of cell-free tumor DNA: one kit without unique molecular identifiers (UMIs) but in technical duplicates and one kit with UMIs. Two reference cell-free DNA samples were used with 0 and $0.13 \%$ tumor allele frequency, respectively. Targeted sequencing was performed to $50,000 \times$ average depth. Illumina's UMI Error Correction Local App was used for aligning and collapsing UMI sequences. Signal noise in UMI- and non-UMI libraries was compared, and effective sequencing depth loss due to the bioinformatic processing was quantified to allow for estimating the required sequencing depth.

\section{KEYWORDS:}

cell-free DNA • low allele frequency • next-generation sequencing • unique molecular identifier (UMI)

Cancer is the second leading cause of death worldwide, with lung cancer being the cancer entity causing the highest number of fatalities [1]. Approximately $40-50 \%$ of patients with non-small-cell lung cancer (NSCLC) are diagnosed with advanced or metastatic disease and are not candidates for curative therapy but only for life-prolonging and palliative treatment. After the exhaustion of standard treatments, some patients may be discussed by molecular tumor boards and included into clinical trials based on their tumor mutations. These mutations are usually revealed by a tissue biopsy, but liquid biopsy is gaining interest because of its ability to reveal tumor mutations in a minimally invasive manner. Furthermore, liquid biopsy can potentially predict treatment response and detect minimal residual disease after cancer surgery. The percentage of cell-free tumor DNA in blood plasma ranges from more than 65\% in advanced patients [2] to $0.1 \%$ or lower in lung cancer patients with less advanced disease [3]. Feasibility, sensitivity/specificity and cost-effectiveness are crucial factors in clinical implementation. Thus, an overall aim must be to supply reliable data to the individual lung cancer patient and, at the same time, to set up the analyses to be simple and inexpensive enough that services may be applied broadly. In this article, we sought to explore these factors for two methods of library preparation and analysis to determine a cost-efficient and robust workflow and to determine the thresholds of artifacts that would confound the detection of low percentages of tumor. We used the Multiplex I cfDNA Reference Standard Set (Horizon Discovery Ltd., Cambridge, UK): the 100\% Multiplex I Wild Type cfDNA Reference Standard (Cat \#HD776) and the $0.1 \%$ Multiplex I cfDNA Reference Standard (Cat \#HD779) with mutated PIK3CA p.E545K (chr3: g.178936091G>A in the hg19 genome version) of $0.13 \%$ allele frequency according to the supplier. From each DNA, relatively inexpensive libraries without UMIs were prepared in duplicates (TruSeq Nano, Cat \#FC-121-4001, Illumina Inc., CA, USA) using a modified protocol for low input and small fragment sizes (160 bp fragments according to Horizon Discovery). In short, modifications to the protocol were the omission of the shearing step due to already fragmented DNA and therefore starting at the end repair step as the entry point with 50 ng of cell-free DNA (cfDNA), SPRIselect bead (SPB) cleanup without size selection ( $2 \times$ vol SPB) and 12 cycles of PCR instead of eight. From 40 ng of each standard, libraries with unique molecular identifier (UMIs) for cfDNA (TruSight Oncology UMI Reagents, Cat \#20024586, Illumina Inc.) were prepared according to protocol, and $500 \mathrm{ng}$ of all six libraries were pooled in equimolar amounts. Targeted capture was done according to the protocol for $x$ Gen hybridization capture of DNA libraries (Integrated DNA Technologies, Inc., Coralville, IA, USA), with custom oligos for coding regions of PIK3CA supplied by Illumina, and with 10 PCR cycles. The captured pool was sequenced with an Illumina NextSeq 500 using $2 \times 150$ bp paired-end reads. We aimed for the raw sequencing depth in the target region to be approximately $50,000 \times$. The sequences were aligned to hg19 using bwa [4] and sorted with samtools [5]. For the settings of all bioinformatic tools used, see Supplementary Table 1. Library type-specific bioinformatic filtering was performed as follows: The TruSight Oncology UMI sequences were aligned and collapsed to consensus sequences using the UMI Error Correction Local App (Illumina Inc.). This software package identifies 'families' of sequence duplicates that are aligned to the same genomic position and that have the same UMI (see Figure 1) and replaces each 'family' of duplicates with a single consensus sequence, resulting in a 'stitched' bam file of aligned, error-corrected consensus sequences, thus reducing the impact of randomly occurring PCR artifacts on the final alignment and variant calling as well as reducing potential sequencing artifacts. The UMls are unique sequences of seven-nucleotides length that are added to each end of a cfDNA fragment during library preparation before PCR is performed. From the raw sequencing files in binary base call (BCL) format, the UMI Error Correction Local App performs base calling, adds the UMI sequences into the sequence names, performs sequence alignment using bwa [4] and samtools [5] and collapses families of sequences that were aligned to the same genomic position and that have the same UMI (see Figure 1) into an error-corrected consensus sequence. This is an automatic process in which two default parameters can be changed: the alignment quality threshold (default: 0 ) and the minimal read family threshold (default: 2). We used the manufacturer's settings, according to standard laboratory practices and International Organization of Standardization recommendations.

For the TruSeq Nano sequences (without UMIs), stringent bioinformatic quality filtering was performed on the raw sequences before alignment to assess whether the signal noise could be reduced compared with standard filtering, that is, to remove sequence instrumentbased artifacts. This stringent filtering was performed by eliminating all paired-end sequences that did not meet a phred-scaled base quality of 32 for each base, using fastp [6].

To test whether the combined analysis of the technical replicates can reduce signal noise in the TruSeq Nano library duplicates, an artificial dataset was created. We thus pooled randomly extracted sequence read-pairs from each of the two TruSeq Nano library duplicates' sequence data files into a single sequence data set. For better comparability between all sequence data sets, we normalized 


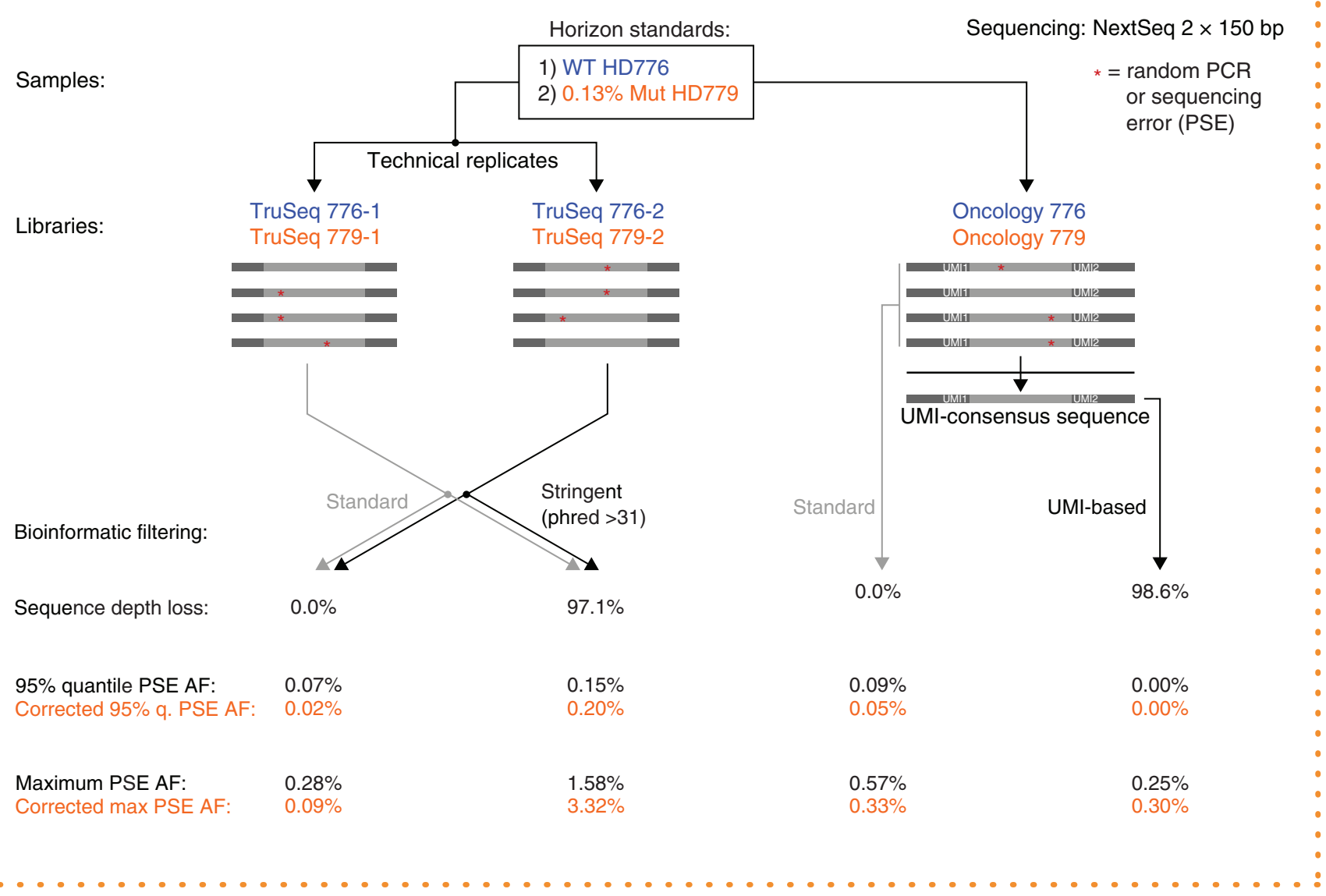

Figure 1. Schematic representation of analysis workflow. Horizon cell-free DNA reference standards HD776 (WT) and HD779 (0.13\% known allelic frequency of g.178936091G >A, hg19) were both prepared with Illumina TruSeq Nano and TruSight Oncology library preparation kits. For TruSight Oncology (with unique molecular identifier [UMIs]) error-corrected consensus sequences were generated from read families and compared with standard filtering. For TruSeq Nano (without UMIs), standard filtering was compared with stringent filtering and to the TruSight Oncology results. The quality metrics for the comparisons were the $95 \%$ quantile of the PCR and sequencing errors' (PSE) allelic frequency (AF) and maximum PSE AF. The PSE AFs were corrected (and rounded to two digits) on the basis of the HD779 standard with its $0.13 \%$ mutation allelic frequency.

the pooled library sequence sets (TruSeq 776-Norm, TruSeq 779-Norm) to have approximately identical sequencing depths. Normalization consisted of random sequence data extraction, alignment, computation of sequence depth in the target region (specifically, at the PIK3CA mutation) and iterative repetition of these steps until the sequencing depth in the target region in the normalized TruSeq Nano libraries was comparable with the respective TruSight Oncology libraries. In theory, this bioinformatic process should reduce the randomly appearing artifacts introduced by the significant amount of PCR cycles in the library preparation because those should appear randomly and independently in technical replicates but should not reduce the artifacts arising from the sequencing instrument.

BAM files of the sequences were deposited at the European Nucleotide Archive under Accession http://www.ebi.ac.uk/ena/data/view/PRJEB39899. GenSearchNGS [7] (PhenoSystems S.A., Braine le Chateau, Belgium) was used to analyze the bam files for low allele frequency variants in joint-variant-calling mode, with and without the option 'ignore duplicate reads'. The 'ignore duplicate reads' option was not used for the analysis of the UMI-based error corrected 'stitched' bam files because the redundant sequences were already collapsed into error-corrected consensus sequences by the UMI Error Correction Local App. The resulting two multisample variant lists (with and without duplicate reads, i.e., redundant sequences) were exported in csv format. Allele frequencies were recomputed from the 'coverage' and 'reads' columns. Lower coverage genomic positions (less than $500 \times$ in the alignments) were filtered. Box plots and density plots of low-allele-frequency PCR and sequencing errors (signal noise) for each library type were plotted in R. The allelic frequency of the signal noise was corrected as follows using the known allelic frequency of the mutation in HD779 versus the sequenced allelic frequency values:

$$
\mathrm{AF}_{\text {corrected }}=\frac{\mathrm{AF}_{\text {uncorrected }} \times 0.13 \%}{A F_{\text {at E545 mutation }}}
$$




\begin{tabular}{|c|c|c|c|c|c|c|}
\hline Type & Library & Filtering $^{\dagger}$ & Mean coverage $\ddagger$ & $\mathbf{N}^{\S}$ & AFI $95 \%$ quantile & AFI max \\
\hline \multirow[t]{10}{*}{ Non-UMI } & TruSeq 776-1 & - & 55472 & 9902 & $0.07 \%$ & $0.28 \%$ \\
\hline & & phred $>31$ & 2160 & 7108 & $0.10 \%$ & $0.79 \%$ \\
\hline & TruSeq 776-2 & - & 52573 & 9902 & $0.07 \%$ & $0.25 \%$ \\
\hline & & phred $>31$ & 1416 & 7108 & $0.12 \%$ & $1.58 \%$ \\
\hline & TruSeq 776-Norm & - & 51340 & 9902 & $0.07 \%$ & $0.25 \%$ \\
\hline & TruSeq 779-1 & - & 47552 & 9902 & $0.07 \%$ & $0.20 \%$ \\
\hline & & phred $>31$ & 789 & 7108 & $0.15 \%$ & $0.77 \%$ \\
\hline & TruSeq 779-2 & - & 57491 & 9902 & $0.07 \%$ & $0.25 \%$ \\
\hline & & phred $>31$ & 1948 & 7108 & $0.10 \%$ & $0.80 \%$ \\
\hline & TruSeq 779-Norm & - & 61746 & 9902 & $0.07 \%$ & $0.22 \%$ \\
\hline \multirow[t]{4}{*}{ UMI } & Oncology 776 & - & 36786 & 9963 & $0.09 \%$ & $0.51 \%$ \\
\hline & & Consensus & 602 & 7141 & $0.00 \%$ & $0.25 \%$ \\
\hline & Oncology 779 & - & 48924 & 9963 & $0.09 \%$ & $0.57 \%$ \\
\hline & & Consensus & 670 & 7141 & $0.00 \%$ & $0.25 \%$ \\
\hline
\end{tabular}

$\dagger$ Filtering: phred $>31$ considered only read-pairs with base quality $>31$ for all bases in the read-pair. Consensus filtering with the IIlumina UMI app collapsed duplicate reads with identical UMls to a consensus read.

$¥$ Mean coverage: considered the entire coding region of PIK3CA but counted only bases with at least phred $>19$.

$\S_{\mathrm{N}}$ : number of observations (genomic positions in PIK3CA with sequencing depth at least $500 \times$ in all libraries of the same type).

IAF: Allele frequency of the PCR and sequencing errors, the computation of which considered only those genomic coordinates with a minimal sequencing depth of $500 \times$ in libraries of the same group (TruSeq unfiltered, TruSeq filtered, Oncology unfiltered, Oncology filtered)

The main results can be summarized as follows (Figure 1).

The allele frequency of PCR and sequencing errors (signal noise) was reproducibly lower than $0.07 \%$ for the four non-UMI libraries in $95 \%$ of the considered genomic positions (Table 1) and exactly $0.00 \%$ for the two UMI libraries in $95 \%$ of positions. However, numerous PCR and sequencing error outliers were detected with higher allele frequencies of up to $0.28 \%$ in the four non-UMI libraries (Figure $2 \mathrm{~A}$ \& Table 1) and up to $0.25 \%$ in the two UMl libraries with consensus filtering (Figure 2D \& Table 1), after ensuring that the effective sequencing depth at each considered position was at least 500 reads. The PCR and sequencing errors' allele frequencies were higher if lower effective sequencing depths were permitted. For the non-UMI libraries, stringent bioinformatic filtering reduced sequencing depths and increased the allele frequency of the PCR and sequencing errors. On the other hand, for the UMI libraries, bioinformatic error correction lowers the allele frequency of the PCR and sequencing errors, despite reducing the effective sequencing depth (Table 1). Of particular note for those who are responsible for the specification and the costs of experiments: in our combination of Illumina and IDT protocols, the mean effective sequencing depth in the PIK3CA target region was reduced 60 -fold by the bioinformatic error-processing of UMI libraries.

The known $0.13 \%$ PIK3CA p.E545K mutation can be used to calibrate the allelic frequencies obtained from the sequencing reads (Supplementary Table 2) and then used to correct the allelic frequency of the PCR and sequencing errors. Table 2 shows the corrected summary values. For the non-UMI libraries, the calibrated signal noise is up to threefold lower than before calibration, indicating high levels of signal noise - probably PCR duplicates - at the PIK3CA mutation site and therefore at all sites. For the UMI libraries after bioinformatic consensus filtering, the signal noise is nearly unchanged after calibration. This is confirmed by the more accurate tumor allele frequency of $0.1 \%$ for the UMI-based error corrected sequences at the PIK3CA site compared with $0.4 \%$ from the libraries without UMIs (Supplementary Table 2).

There are also three secondary results that may be of interest to a laboratory: first, when UMI-based error correction bioinformatics are used, the conventional quality criterion of a well-balanced ratio of forward-reverse sequences is not applicable (Supplementary Figure 1). This is a purely bioinformatic result after the UMI-based error correction because the original sequences are well balanced before error correction. Second, in the non-UMI libraries, bioinformatic removal of redundant sequences (deduplication) was detrimental for the signal-to-noise ratio because it reproducibly amplified the signal noise (Supplementary Table 3 ) but reduced the allele frequency of the true mutation (Supplementary Table 2). Third, the PCR and sequencing errors' allele frequencies were not improved in our normalization sub-experiment (TruSeq 776-Norm, TruSeq 779-Norm), see Table 1 \& Supplementary Table 3, suggesting that PCR error was not the dominant contributor to the total sequencing error.

In conclusion, the mean loss of effective sequence depth in the UMI libraries after bioinformatic error correction is on the order of $98.5 \%$, requiring 60 -fold deeper sequencing than for conventional non-UMI libraries. This problem is due to the small amounts of cfDNA that are available from a human blood sample and the resulting 25 cycles of PCR that were needed in the UMI-based protocol. By comparison, the original report by Schmitt and colleagues [8] on UMI-based error correction demonstrated its principle using $3 \mu \mathrm{g}$ of input 


\section{TruSeq nano library (without UMI)}

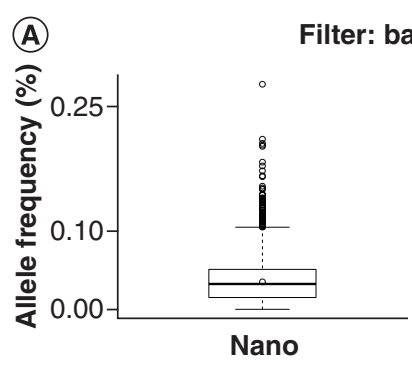

(B)

Filter: base quality $>\mathbf{3 1}$ for every base of every read
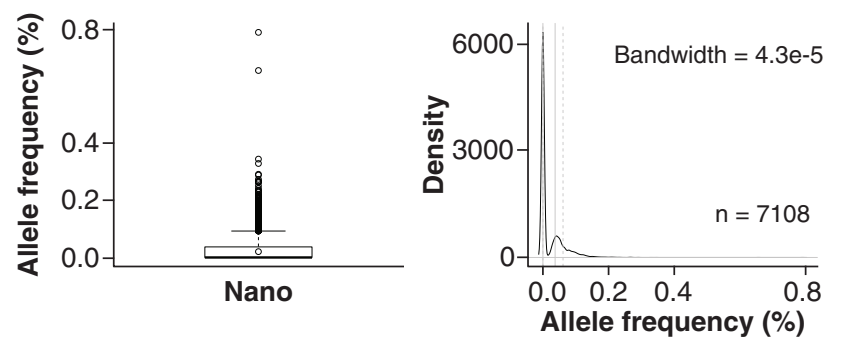

TruSight oncology library (with UMI)

(C) Without UMI-based read-deduplication
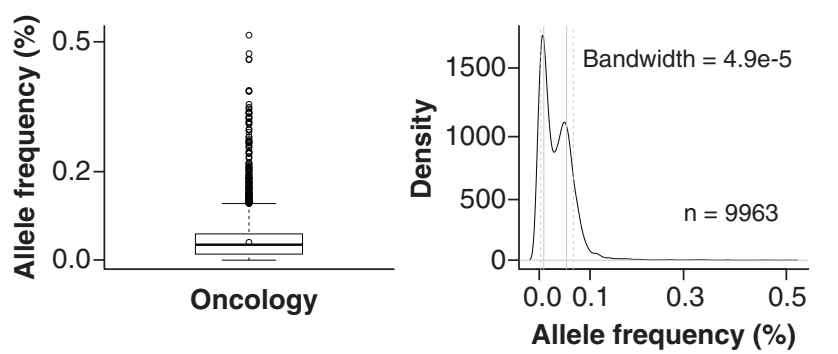

(D) With UMI-based dedup and error-correction

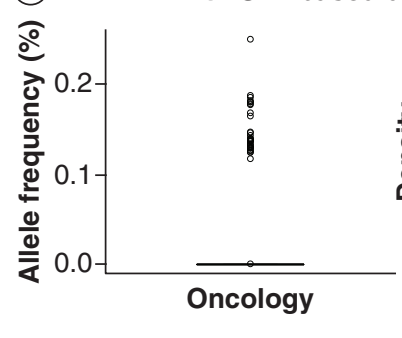

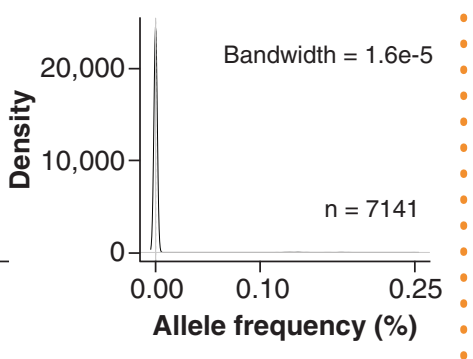

Figure 2. Allele frequency distributions of noncalibrated PCR and sequencing errors (signal noise) depending on kit and bioinformatic processing. The plots show the signal-noise allele frequency (AF) ranges for the wild-type Horizon standard HD776, from targeted sequencing of the PIK3CAa coding regions. The AFs are the 'as-is' AFs, that is, without the HD779 mutation-based correction/calibration. The density plots include the $12.5 \%, 25 \%, 75 \%$ and $87.5 \%$ quantiles. (A) Non-unique molecular identifier (UMI) library type TruSeq Nano, where only base calls with base quality $>19$ were considered. Without calibration, the max AF is $0.28 \%$; with calibration it is only $0.09 \%$. The density plot shows a bimodal distribution of allele frequencies, which might be explained by a large number of high-quality base calls with small AFs (left peak) and a slightly smaller number of lower quality base calls with higher AFs (right peak), because the right peak nearly disappears when stringent base quality filtering is applied (see panel B). The right peak (higher $\mathrm{AFs}$ ) is not due to sequence duplication because a similar bimodal distribution can be seen after filtering duplicates or redundant sequences (data not shown). (B) Non-UMI library type TruSeq Nano, where only highest quality read-pairs (all base qualities $>31$ ) were used for alignment and variant calling. In the density plot, the majority of AFs are exactly zero, and a small peak can be seen at $0.05 \% \mathrm{AF}$. Of note, the max AF has risen from $0.28 \%$ to $0.8 \%$, which is explained by the lower sequencing depth after stringent filtering. (C) UMI-library type TruSight Oncology, where conventional alignment was performed without utilizing the UMIs for error correction or read deduplication. (D) UMI-library type TruSight Oncology, where the Illumina app was used for UMI-based deduplication and error correction.

DNA and only 18-20 PCR cycles. We would obtain $3 \mu \mathrm{g}$ of cfDNA from a hypothetical 1-I blood draw, which would have detrimental health effects on every cancer patient except for the extremely rare elephant with cancer. A 10-ml blood draw from a human patient will result in approximately $4-5 \mathrm{ml}$ of plasma and $40-50 \mathrm{ng}$ of cfDNA. On the other side, Illumina sequencers need a certain loading concentration of library, and a high number of PCR cycles is required in the library preparation of low-input samples. Therefore, the highly duplicated cfDNA molecules will be collapsed to a small fraction after bioinformatic error correction. According to the product information, an increase in sequencing depth will probably not greatly increase the sequence depth of UMI-collapsed consensus reads. Special care should be given to optimization processes in the lab, especially the recovery in the cleanup steps, as well as hybridization and enrichment. Non-UMI libraries have inherently higher signal noise and are less accurate with respect to allele frequency quantification. This means that the allele frequencies of mutations detected with non-UMI-libraries should be calibrated with known tumor allele frequencies by including reference DNA samples into each non-UMI NGS run. Regardless of library type, technical replication is recommended to identify whether variants with low allele frequencies are PCR and sequencing errors. Ideally, control DNAs should be included, such as the Horizon cfDNA set used here.

In summary, this article has identified advantages and drawbacks of two methods for detecting low-frequency alleles in liquid biopsies. At present, applications in cancer care are limited due to high cost. To provide real-life, broad clinical benefit to cancer patients, more optimizations that ensure reliable results in a cost-efficient workflow are crucial.

\section{Future perspective}

Currently, cell-free DNA-based cancer tests are offered routinely in the USA for privately insured patients; in many other countries, they are available for patients taking part in research studies. Molecular tumor boards (MTBs) are being initiated in cancer hospitals in many 


\begin{tabular}{|c|c|c|c|c|c|c|}
\hline Type & Library & Filtering ${ }^{\dagger}$ & Mean coverage $\mathrm{e}^{\ddagger}$ & $\mathbf{N}^{\S}$ & AFI $95 \%$ quantile & $\mathrm{AF}^{\mathbb{I}} \max$ \\
\hline \multirow[t]{10}{*}{ Non-UMI } & TruSeq 776-1 & - & 55472 & 9902 & $0.02 \%$ & $0.09 \%$ \\
\hline & & phred $>31$ & 2160 & 7108 & $0.20 \%$ & $1.65 \%$ \\
\hline & TruSeq 776-2 & - & 52573 & 9902 & $0.02 \%$ & $0.08 \%$ \\
\hline & & phred $>31$ & 1416 & 7108 & $0.24 \%$ & $3.32 \%$ \\
\hline & TruSeq 776-Norm & - & 51340 & 9902 & $0.02 \%$ & $0.08 \%$ \\
\hline & TruSeq 779-1 & - & 47552 & 9902 & $0.02 \%$ & $0.06 \%$ \\
\hline & & phred $>31$ & 789 & 7108 & $0.30 \%$ & $1.62 \%$ \\
\hline & TruSeq 779-2 & - & 57491 & 9902 & $0.02 \%$ & $0.08 \%$ \\
\hline & & phred $>31$ & 1948 & 7108 & $0.21 \%$ & $1.67 \%$ \\
\hline & TruSeq 779-Norm & - & 61746 & 9902 & $0.02 \%$ & $0.07 \%$ \\
\hline \multirow[t]{4}{*}{ UMI } & Oncology 776 & - & 36786 & 9963 & $0.05 \%$ & $0.30 \%$ \\
\hline & & Consensus & 602 & 7141 & $0.00 \%$ & $0.30 \%$ \\
\hline & Oncology 779 & - & 48924 & 9963 & $0.05 \%$ & $0.33 \%$ \\
\hline & & Consensus & 670 & 7141 & $0.00 \%$ & $0.30 \%$ \\
\hline
\end{tabular}

${ }^{\dagger}$ Filtering: phred $>31$ filtering considered only read-pairs with base quality $>31$ for all bases in the read-pair. Consensus filtering with the Illumina UMI app collapsed duplicate reads with identical UMIs to a consensus read.

$\ddagger$ Mean depth: considered the entire coding region of PIK3CA but counted only bases with at least phred $>19$.

$\S \mathrm{N}$ : number of observations (genomic positions in PIK3CA with sequencing depth at least $500 \times$ in all libraries of the same type).

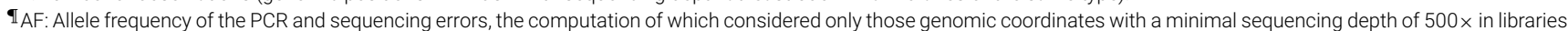
of the same group (TruSeq unfiltered, TruSeq filtered, Oncology unfiltered, Oncology filtered). The AF values in this table have been corrected using the known PIK3CA AF of $0.13 \%$ versus the sequenced AF in the HD779 DNA. The two methods with the smallest PCR and sequencing errors' AFs are highlighted in bold: non-UMI TruSeq libraries without filtering or UMI Oncology libraries with consensus filtering.

countries, following the model established by leading cancer centers in the USA. The MTBs discuss individual patient cases for potential individualized treatment after exhaustion of standard treatments, such as for the choice of a suitable clinical trial. An ever-increasing portfolio of drugs and drug combinations is being tested on cancer patients in clinical trials, some with mutation-based inclusion criteria. Tissue biopsy has been the gold standard for mutation detection, but the clear advantages of liquid biopsy in longitudinal monitoring of resistance mechanisms with the representativeness of all tumor sites will have a major impact in the field of individualized treatment in the coming years. From our past experience, the cost of high-fidelity blood-based cell-free DNA cancer tests is likely to drop to a 10th of the current costs, allowing their widespread use, for example, for monitoring the therapy response of advanced-stage cancer patients and detecting minimal residual disease after cancer surgery. Many of the clinical guidelines will be updated with recommendations for cell-free DNA testing, based on currently ongoing clinical studies that are exploring the clinical utility of these tests. In approximately 10 years, cost-benefit analyses by public health systems and their professional societies are likely to be completed in all EU countries, defining the indications for which patients with a public health insurance will be entitled to receive free high-fidelity cell-free DNA-based cancer tests. It seems most likely that the methods race will be won by UMI-based sequencing and error-correction methods, using a highly automated high-fidelity sequencing platform that will have a hundred times more sequence output than the current largest instruments.

\section{Supplementary data}

To view the supplementary data that accompany this paper please visit the journal website at: www.future-science.com/doi/suppl/10. 2144/btn-2020-0124

\section{Author contributions}

M Forster and J Fuß designed the study. MS Frank, J Fuß, TA Steiert, G Streleckiene, J Gehl and M Forster wrote the manuscript. J Fuß organized lab work. G Streleckiene, TA Steiert and M Forster analyzed the data. MS Frank and J Gehl interpreted the data with respect to prospective application in the clinical care for lung cancer patients. J Fuß drafted the graphical abstract.

\section{Acknowledgments}

Regina Fredrik performed lab work. Andre Franke and Philip Rosenstiel provided lab facilities. 


\section{Financial \& competing interests disclosure}

This work was supported by Changing Cancer Care, funded by Interreg Deutschland-Denmark supported by the European Regional Development Fund. This work was also supported by the Research Council of Lithuania, grant no. 09.3.3-LMT-K-712-01-0130. The authors have no other relevant affiliations or financial involvement with any organization or entity with a financial interest in or financial conflict with the subject matter or materials discussed in the manuscript apart from those disclosed.

No writing assistance was utilized in the production of this manuscript.

\section{Open access}

This work is licensed under the Attribution-NonCommercial-NoDerivatives 4.0 Unported License. To view a copy of this license, visit http://creativecommons.org/licenses/by-nc-nd/4.0/

\section{References}

Papers of special note have been highlighted as: $\bullet \bullet$ of considerable interest

1. American Cancer Society. Cancer facts \& figures 2020. American Cancer Society, GA, USA (2020). www.cancer.org/research/cancerfactsstatistics/cancerfactsfigures2020/index

2. Hendricks A, Rosenstiel P, Hinz S et al. Rapid response of stage IV colorectal cancer with APC/TP53/KRAS mutations to FOLFIRI and bevacizumab combination chemotherapy: a case report of use of liquid biopsy. BMC Med. Genet. 21(1), 3 (2020).

-. Clinical case report demonstrating the clinical utility of cell-free DNA testing in a stage IV cancer patient.

3. Abbosh C, Birkbak NJ, Wilson GA et al. Phylogenetic ctDNA analysis depicts early-stage lung cancer evolution. Nature 545(7655), 446-451 (2017).

-• Lung cancer study report on sequencing low-input amounts of cell-free DNA with non-UMI sequencing libraries in combination with calibration DNA samples.

4. Li H, Durbin R. Fast and accurate short read alignment with Burrows-Wheeler transform. Bioinformatics 25(14), 1754-1760 (2009).

5. Li H, Handsaker B, Wysoker A et al. The Sequence Alignment/Map format and SAMtools. Bioinformatics 25(16), 2078-2079 (2009).

6. Chen S, Zhou Y, Chen Y, Gu J. fastp: an ultra-fast all-in-one FASTQ preprocessor. Bioinformatics 34(17), i884-i890 (2018).

7. Wolf B, Kuonen P, Dandekar T, Atlan D. DNAseq workflow in a diagnostic context and an example of a user friendly implementation. Biomed. Res. Int. 2015, 403497 (2015).

8. Schmitt MW, Kennedy SR, Salk JJ et al. Detection of ultra-rare mutations by next-generation sequencing. Proc. Natl Acad. Sci. USA 109(36), 14508-14513 (2012).

-. Report on the theoretical error rates of the duplex sequencing method using UMls and error correction, demonstrated in a laboratory experiment using a high-input amount of $3 \mu \mathrm{g}$ DNA and only 18-20 PCR cycles. 